Proceedings of the International School and Conference on Optics and Optical Materials, ISCOM07, Belgrade, Serbia, September 3-7, 2007

\title{
Holographic Fabrication of Periodic Microstructures in Dichromated Pullulan
}

\author{
S. Savić-Šević* , D. Pantelić, R. Gajić and G. Isić \\ Institute of Physics, Pregrevica 118, 11080 Belgrade, Serbia \\ Photonic crystal structures are fabricated in dichromated pullulan by \\ the holographic technique. Relief structures of photonic lattices with rectan- \\ gular, rhombic, and hexagonal arrays of peaks and holes are obtained. The \\ structures have periodicities of the order $1.1 \mu \mathrm{m}$ and depth of about $50 \mathrm{~nm}$.
}

PACS numbers: 42.40.--i, 42.70.Ln, 42.70.Qs

\section{Introduction}

One way to create photonic crystal structures is realized by holography [1-4]. In this study dual-beam multiple exposure technique is used to generate various lattice structures. In the dual-beam process, the photonic crystal structure is obtained by rotating the holographic recording material between exposures. A range of lattice structures can be obtained by varying the angle between two beams, and the rotation angle of holographic material.

Previously, photonic structures have been recorded also in low-refractive index materials such as photoresists [4]. The holographic material used in this study is pullulan, doped with dichromate $[5,6]$. Pullulan is a biopolymer with linear polysaccharide structure.

\section{Periodic microstructures fabrication}

Dichromate-sensitized pullulan samples were prepared by mixing $8 \%$ acqueous solution of pullulan and $50 \%$ ammonium dichromate by weight of pullulan. The solution was subsequently coated onto clean glass slides in a horizontal position and the film was dried under normal laboratory conditions. The thickness of the dried layer was approximately $10 \mu \mathrm{m}$.

A single-frequency, diode pumped Nd:YAG laser, at $532 \mathrm{~nm}$, is used for exposure (200 $\mathrm{mW}$ laser power). Linearly polarized, laser beam is split into two

*corresponding author; e-mail: savic@phy.bg.ac.yu 
equal power beams (to obtain the highest contrast in fringe intensity) and the beam interference pattern is recorded. He-Ne laser operating at $632.8 \mathrm{~nm}$ was used for real-time monitoring of diffraction efficiency. By proper choice of rotation angles different structures can be generated [7]. Double and triple exposures, to the same interference pattern of dichromated pullulan, were used.

The exposed plates were, first, chemically processed with a mixture of $60 \%$ isopropanol and $40 \%$ water for 120 seconds, and then immersed for 30 seconds in the pure isopropanol. After development in mixture of water and isopropanol unexposed regions are washed out, and an intensity light pattern is converted into a relief structure.

The structures were characterized by atomic force microscope (AFM) needle sensor on the TwinSnom system.

Relief structure of photonic lattice with rectangular array of peaks and holes was obtained by rotating the material for $90^{\circ}$, between exposures. Figure 1 shows an AFM image of the lattice structure.
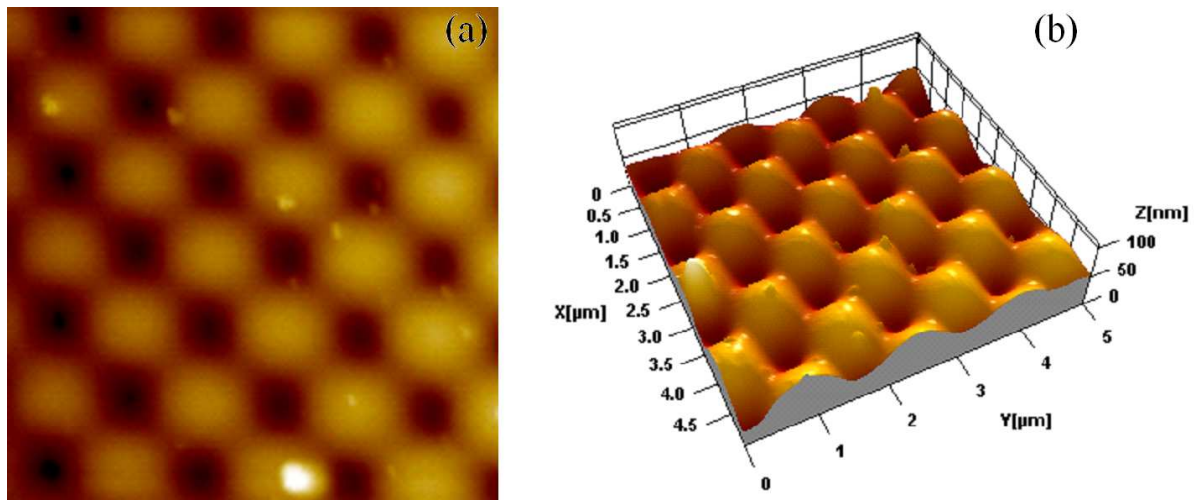

Fig. 1. AFM images of a rectangular photonic crystal structure. (a) Top view, (b) 3D view of the same sample.

A rhombic photonic crystal lattice was similarly produced by rotating the material for $45^{\circ}$, Fig. 2 .

A hexagonal photonic crystal structure was produced using three exposures with holographic material rotated by $60^{\circ}$ and $120^{\circ}$, Fig. 3 .

The structures have periodicities of the order $1.1 \mu \mathrm{m}$ and depth about $50 \mathrm{~nm}$.

It could be seen that the structure from Fig. 3 has smaller depth contrast, compared to structures shown in Figs. 1 and 2. While structures from Figs. 1 and 2 were produced with two exposures (only one intermediate rotation), the structure shown in Fig. 3 was obtained with three exposures, so the depth contrast was decreased because of cumulative angle errors of two intermediate rotations. However, by more precise control of rotation angle of the holographic recording material between exposures the depth contrast could be improved. 

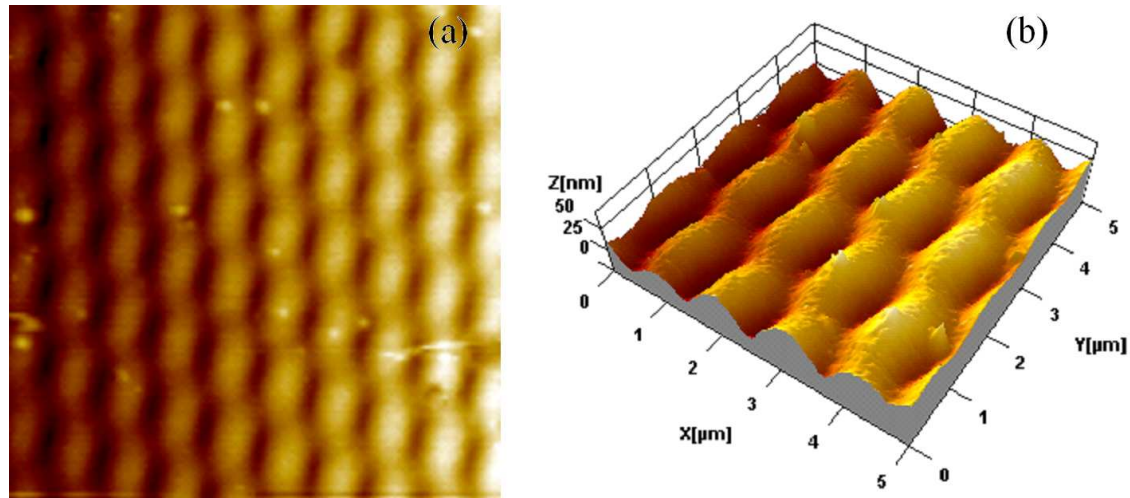

Fig. 2. AFM images of a rhombic photonic crystal structure. (a) Top view, (b) 3D view of the same sample.
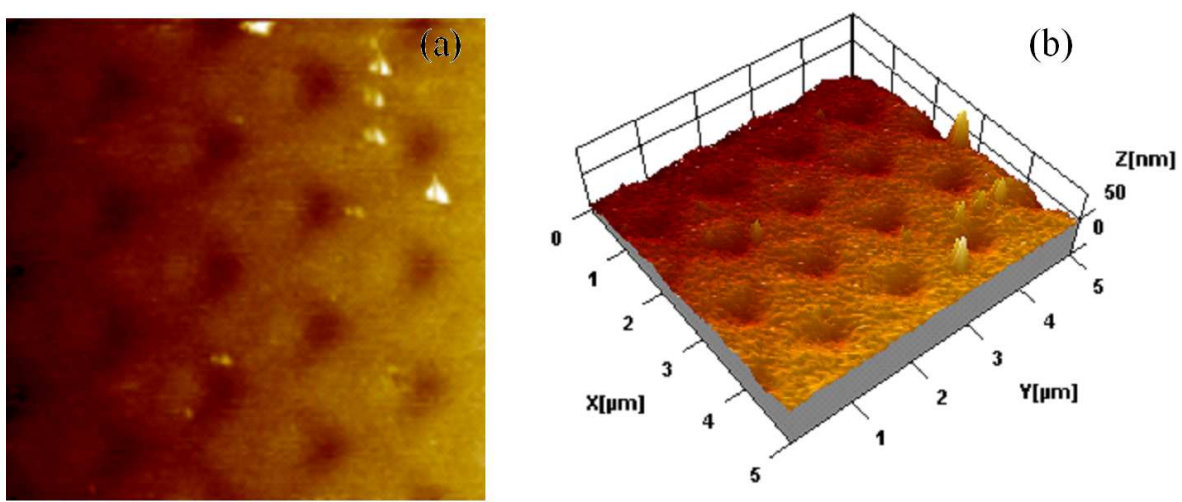

Fig. 3. AFM images of a hexagonal photonic crystal structure. (a) Top view, (b) 3D view of the same sample.

\section{Conclusion}

We have fabricated photonic crystal structures in a dichromate-sensitized pullulan by holography. Dual-beam multiple exposure technique is used. Our microstructures, although with low refractive index, may be used as templates for the construction (development) of photonic crystal with higher refractive index contrast for complete band gaps.

\section{Acknowledgments}

Research was performed under the contract 141003 funded by the Ministry of the Science and Environmental Protection of Serbia. 


\section{References}

1] M. Campbell, D.N. Sharp, M. Harrison, R.G. Denning, A.J. Turberfield, Nature 404, 53 (2000)

[2] Y.K. Pang, J.C.W. Lee, C.T. Ho, W.Y. Tam, Opt. Express 14, 9013 (2006).

[3] S. Pissadakis, C. Pappas, Opt. Express 15, 4296 (2007).

[4] T. Kondo, S. Matsuo, S. Juodkazis, V. Mizeikis, Appl. Phys. Lett. 82, 2758 (2003).

[5] D. Pantelić, S. Savić, D. Jakovljević, Opt. Lett. 23, 807 (1998).

[6] S. Savić-Šević, D. Pantelić, Opt. Express 13, 2747 (2005).

[7] R.C. Gautheir, K.W. Mnaymneh, Opt. Laser Technol. 36, 625 (2004). 